\title{
PCOS serum-derived exosomal miR-27a-5p stimulates endometrial cancer cells migration and invasion
}

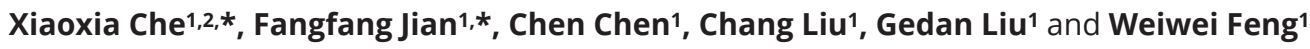 \\ 1Department of Obstetrics \& Gynecology, Ruijin Hospital, School of Medicine, Shanghai Jiao Tong University, Shanghai, China \\ 2Obstetrics and Gynecology Hospital, Fudan University, Shanghai, China
}

Correspondence should be addressed to W Feng: fww12066@rjh.com.cn

*(X Che and F Jian contributed equally to this work)

\begin{abstract}
Polycystic ovary syndrome (PCOS) is the most common endocrine disorder among reproductive-age women. Women with PCOS have a 2.7-fold increased risk for developing endometrial cancer (EC). This study was performed to investigate the potential stimulatory effects of serum exosomes isolated from patients with PCOS on EC cell lines and to explore the underlying mechanism. EC cell lines exposed to exosomes derived from PCOS patients serum exhibited an enhanced migration and invasion phenotype. Next, sequence-based analysis of exosomal miRNA was conducted to screen the differentially expressed miRNAs in serum exosomes from PCOS patients and normal controls. The levels of 55 mature miRNAs significantly differed in serum exosomes from PCOS patients compared with those from normal controls. Real-time PCR was used to verify the expression of eight of these miRNAs, among which miR-27a-5p was the most significantly elevated in PCOS patients serum exosomes. The role of miR-27a-5p in EC migration and invasion was further investigated via miR-27a-5p mimics or inhibitor transfection in Ishikawa and HEC-1A EC cell lines. In addition, the SMAD4 gene was identified as the target of miR-27a-5p by several target prediction databases and was validated by a luciferase assay. SMAD4 mRNA and protein levels were downregulated in EC cells transfected with the miR-27a-5p mimics, but upregulated in cells transfected with the miR-27a-5p inhibitor. Furthermore, in vitro experiments results confirmed that miR-27a-5p prohibited migration and invasion via SMAD4 downregulation. Thus, serum exosomal miR-27a-5p may play a role in EC development in PCOS patients.
\end{abstract}

\author{
Key Words \\ - PCOS \\ - exosome \\ - miR-27a-5p \\ - endometrial cancer
}

\section{Introduction}

Polycystic ovary syndrome (PCOS), characterized by oligo/anovulation, hyperandrogenism and polycysticappearing ovaries, is the most common endocrine disorder among reproductive-age women. Complex interrelationships between endometrial cancer (EC) and
PCOS have been recognized for several years, and multiple risk factors, including diabetes, obesity, anovulation and nulliparity, are involved (Papaioannou \& Tzafettas 2010). Understanding the molecular mechanism of EC occurrence in PCOS patients is of major clinical importance. Recent 
studies have revealed that exosomes can carry and deliver miRNAs to recipient cells (Valadi et al. 2007).

miRNAs are small single-stranded, conserved noncoding RNAs of approximately 22 nucleotides that are derived form endogenous chromosomes. Its modulation in post-transcriptional gene expression is binding imperfectly to the 3'untranslated regions (3'UTRs) of their target mRNAs (Bartel 2004, Ha \& Kim 2014). Recent studies have shown that miRNAs can modulate various cellular processes including cell development, differentiation, proliferation, and metabolism. Altered miRNA expression also plays an essential role in various types of cancers, such as EC, prostate cancer, and colon cancer, and may critically affect multiple steps in tumor progression, including aberrant growth and proliferation, cell death resistance, invasion, and metastasis to distant organs (Yanokura et al. 2015, Kumar \& Lupold 2016, Wu et al. 2018).

Exosomes are membrane-enclosed vesicles of endosomal origin with sizes ranging from 30 to $100 \mathrm{~nm}$ that contain various proteins and lipids as well as various types of nucleic acids, including DNA, RNA, and miRNAs (Liu et al. 2015). Recently, extracellular vesicles have been described to swarm the cancer microenvironment, participating in events such as tumor-stroma and cancerassociated fibroblast (CAF) communication (Patras \& Banciu 2019). Most importantly, exosomes derived from the serum or urine of cancer patients have been proven to be reliable markers for cancer diagnosis (Huang et al. 2015, Melo et al. 2015). Lazar et al. (2016) proposed a novel mechanism linking obesity and cancer. This group proved that adipose tissue-derived exosomes containing fatty acid oxidation products promoted melanoma aggressiveness. In addition, Li et al. (2019a) found that CAF-mediated EC progression was related to the loss of miR-148b in the CAF-derived exosomes. Therefore, we speculated that the serum exosomes from PCOS patients might deliver miRNAs to the endometrium and promote EC progression. The identification of serum biomarkers participating in EC formation that could be used for early intervention for PCOS patients would be highly valuable.

Herein, we show that serum derived exosomal miR-27a-5p from PCOS patients can be transferred to EC cell lines and thereby promote EC cells migration and invasion by targeting SMAD4. Therefore, miR-27a-5p from circulating exosomes from PCOS patients may be used as a blood-based biomarker for the prediction of EC.

\section{Materials and methods}

\section{Study participants}

The present study was approved by the Ethics Committee of Shanghai Jiaotong University. All participants signed an informed consent form. Serum obtained from four normal women served as the control group, while serum obtained between the2ndand5th day of themenstrualcyclefrom four patients with PCOS according to the Rotterdam standard served as the experimental group. Exosomes derived from the above two groups were used for experimental assays and exosomal miRNA sequencing. To verify the miRNA sequencing data, we obtained additional exosomes from ten normal controls and 30 PCOS patients. According to the specific Rotterdam criteria, the samples comprised $20 \%(6 / 30)$ phenotype A (polycystic ovaries+ovulatory dysfunction + hyperandrogenism), 30\% (9/30) phenotype B (ovulatory + hyperandrogenism), 0\% (0/30) phenotype C (polycystic ovaries + hyperandrogenism) and 50\% (15/30) phenotype D (polycystic ovaries + ovulatory dysfunction) as detailed in Fig. 1. In addition, the four PCOS patients used for serum exosomal miRNA sequencing were all phenotype A.

\section{Cell culture}

Two human EC cell lines (HEC-1A and Ishikawa) and the $293 \mathrm{~T}$ cell line were obtained from the Chinese Academy of Sciences (www.cellbank.org.cn.). STR analysis

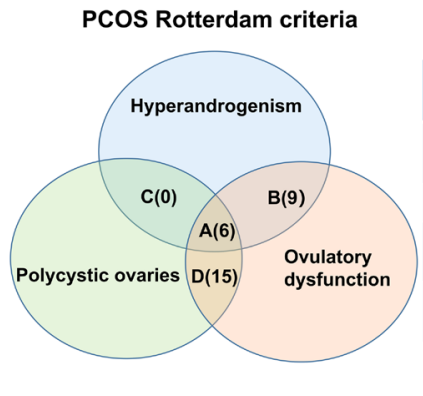

https://jme.bioscientifica.com https://doi.org/10.1530/JME-19-0159

\begin{tabular}{|c|c|c|c|}
\hline Phenotype & Rotterdam criteria & Number & Percent(\%) \\
\hline A & $\begin{array}{l}\text { Polycystic ovaries + Ovulatory } \\
\text { dysfunction + Hyperandrogenism }\end{array}$ & 6 & 20 \\
\hline B & Ovulatory + Hyperandrogenism & 9 & 30 \\
\hline C & $\begin{array}{l}\text { Polycystic ovaries + } \\
\text { Hyperandrogenism }\end{array}$ & 0 & 0 \\
\hline D & $\begin{array}{l}\text { Polycystic ovaries + Ovulatory } \\
\text { dysfunction }\end{array}$ & 15 & 50 \\
\hline
\end{tabular}

(c) 2020 Society for Endocrinology Published by Bioscientifica Ltd. Printed in Great Britain
Figure 1

Ovarian phenotype (in \%) for the PCOS subjects according to the specific Rotterdam criteria in the study. The samples comprised $20 \%(6 / 30)$ phenotype A (polycystic ovaries + ovulatory dysfunction + hyperandrogenism), 30\% (9/30) phenotype B (ovulatory + hyperandrogenism), 0\% (0/30) phenotype $C$ (polycystic ovaries + hyperandrogenism), and 50\% (15/30) phenotype D (polycystic ovaries + ovulatory dysfunction). A full colour version of this figure is available at https://doi.org/10.1530/JME-19-0159. 
certificates were included with all cell lines. HEC-1A cells were maintained in McCoy's 5 A medium, and Ishikawa and $293 \mathrm{~T}$ cells were maintained in DMEM; all media were supplemented with $10 \%$ fetal bovine serum (FBS, Gibco Inc.), $100 \mu \mathrm{g} / \mathrm{mL}$ penicillin/streptomycin and $2 \mathrm{mM}$ L-glutamine. Cells were cultured in a humidified incubator at $37^{\circ} \mathrm{C}$ and $5 \% \mathrm{CO}_{2}$ and tested negative for mycoplasma contamination.

\section{Exosome isolation and quantification}

To isolate exosomes, serum was filtered through $0.22 \mu \mathrm{M}$ filters. Exosomes were isolated using a Qiagen exoEasy Maxi Kit (Qiagen, GM) according to the manufacturer's instructions. Exosomes were characterized by transmission electron microscopy (JEOL Ltd., Tokyo, Japan), NanoSight analysis (Malvern Instruments, Orsay, France), and Western blot analysis (for CD63, HSG101, Alix, and Calnexin).

\section{Transwell migration, invasion, and wound- healing assays}

Transwell migration and invasion assays were conducted in Transwell chambers in a 24-well plate precoated with (for invasion assays) or without (for migration assays) Matrigel (BD BioCoat). The cell suspension was placed into the top chamber of each insert. Transwell chambers with upper and lower culture compartments were separated by polycarbonate membranes with an 8 - $\mu \mathrm{m}$ pore size.

Table 1 Primer sequences used in the study.

\begin{tabular}{|c|c|}
\hline Gene & Primer sequence $\left(5^{\prime}-3^{\prime}\right)$ \\
\hline miR-27a-5p & $\begin{array}{l}\text { F: GCGGCGGAGGGCTAGCTGCTTG } \\
\text { R: ATCCAGTGCAGGGTCCGAGG }\end{array}$ \\
\hline miR-590-3p & $\begin{array}{l}\text { F: GCGTAATTTTATGTATAAGC } \\
\text { R: GTATCCAGTGCGTGTCGTGGAGT }\end{array}$ \\
\hline miR-375-3p & $\begin{array}{l}\text { F: CGCCGTTTGTTCGTTCGGCT } \\
\text { R: CAGCCACAAAAGAGCACAAT }\end{array}$ \\
\hline miR-19b-3p & $\begin{array}{l}\text { F: TGATAATTAGCAAGCAGGATTA } \\
\text { R: ACCAACATTACGCGGCATCATTA }\end{array}$ \\
\hline SMAD4 & $\begin{array}{l}\text { F: CCACCAAGTAATCGTCATCG } \\
\text { R: TGCTAGCATTAGACTCAGATGGG }\end{array}$ \\
\hline GAPDH & $\begin{array}{l}\text { F: ACAGTCAGCCGCATCTTCTT } \\
\text { R: GACAAGCTTCCCGTTCTCAG }\end{array}$ \\
\hline U6 & $\begin{array}{l}\text { F: CGCTTCACGAATTTGCGTGTCAT } \\
\text { R: GCTTCGGCAGCACATATACTAAAAT }\end{array}$ \\
\hline miR-27a-5p mimics & $\begin{array}{l}\text { F: AGGGCUUAGCUGCUUGUGAGCA } \\
\text { R: UGCUCACAAGCAGCUAAGCCCU }\end{array}$ \\
\hline miR ON mimics NC & $\begin{array}{l}\text { F: UUUGUACUACACAAAAGUACUG } \\
\text { R: CAGUACUUUUGUGUAGUACAAA }\end{array}$ \\
\hline $\begin{array}{l}\text { miR-27a-5p inhibitor } \\
\text { miR OFF inhibitor NC }\end{array}$ & $\begin{array}{l}\text { UGCUCACAAGCAGCUAAGCCCU } \\
\text { CAGUACUUUUGUGUAGUACAAA }\end{array}$ \\
\hline
\end{tabular}

Seven hundred microliters of medium containing 10\% FBS was added to the bottom chambers. Two hundred microliters of medium containing cells with or without exosomes was added into the upper chambers. After $24 \mathrm{~h}$, the migrated or invaded cells on the bottom surface were fixed with $4 \%$ paraformaldehyde and stained with a $0.1 \%$ crystal violet solution. Migrated and invaded cells were counted in five randomly selected fields. The experiments were repeated independently in triplicate.

A real-time cell migration assay (RTCA) was conducted with the xCELLigence system (Agilent). This system was used to assess cell migration in real time by measuring electrical impedance, as detailed in a previous study (Irelan et al. 2011). Ishikawa and HEC-1A cells (at a density of 10,000 cells) were plated in serum-free medium containing exosomes from normal controls or PCOS patients. The cell index was normalized to that of cells in the wells at time point 0 following attachment. Cell migration, as measured according to the cell index, was monitored for several days.

For the wound-healing assay, Ishikawa and HEC-1A cells treated with or without exosomes/miR-27a-5p mimics were seeded into six-well plates and allowed to grow to 95\% confluence. A single scratch wound was created, and the cells were washed gently to remove cell debris. Images were acquired by phase contrast microscopy at 0 and $24 \mathrm{~h}$ after wounding.

\section{Immunofluorescence (IF)}

Ishikawa and HEC- $1 \mathrm{~A}$ cells were seeded $\left(10 \times 10^{4}\right.$ cells/well $)$ in a $35-\mathrm{mm}$ dish with a $10-\mathrm{mm}$ bottom well. The cells were allowed to proliferate in standard media (DMEM and McCoy's 5A medium supplemented with 10\% FBS (Gibco Inc.), $100 \mu \mathrm{g} / \mathrm{mL}$ penicillin/streptomycin and $2 \mathrm{mM}$ L-glutamine in a humidified incubator at $37^{\circ} \mathrm{C}$ and $5 \% \mathrm{CO}_{2}$ ) to $60 \%$ confluence overnight. Then, we exposed PKH67-labeled exosomes $(25 \mu \mathrm{g} / \mathrm{mL})$ to EC cells for 24 h. Next we fixed the cells using $4 \%$ paraformaldehyde, stained nuclei with DAPI and examined the cells by confocal microscopy (Leica).

\section{RNA extraction, quantification and quantitative real-time polymerase chain reaction (q-PCR)}

RNA was extracted from isolated exosomes using a miRNeasy Micro Kit (Qiagen) according to the manufacturer's instructions. For analysis of SMAD4 mRNA expression, a qScript cDNA Synthesis Kit (Quanta) with $1000 \mathrm{ng}$ of tRNA was used. For miRNA expression analysis, 
Table 2 Parameters of PCOS patients and normal controls used for exosomal miRNA sequencing.

\begin{tabular}{l} 
Parameter \\
\hline Age (years) \\
Body mass index $\left(\mathrm{kg} / \mathrm{m}^{2}\right)$ \\
FSH $(\mathrm{mlU} / \mathrm{mL})$ \\
$\mathrm{LH}(\mathrm{mlU} / \mathrm{mL})$ \\
AMH $(\mathrm{ng} / \mathrm{mL} / 21-30$ years) \\
$\mathrm{RPL}(\mathrm{ng} / \mathrm{mL})$ \\
E2 $(\mathrm{pg} / \mathrm{mL})$ \\
Progesterone $(\mathrm{ng} / \mathrm{mL})$ \\
Testosterone $(\mathrm{ng} / \mathrm{mL})$ \\
Triglicerydes $(\mathrm{mmol} / \mathrm{L})$ \\
Insulin resistance $(\mu \mathrm{lU} / \mathrm{mL})$ \\
Fasting blood glucose $(\mathrm{mmol} / \mathrm{L})$ \\
\hline
\end{tabular}

\begin{tabular}{l}
\hline Reference range \\
\hline \\
$3.3-7.9$ \\
$2.0-12.0$ \\
$3.31-7.98$ \\
$3.5-24.2$ \\
$18-195$ \\
$0.2-1.2$ \\
$0.15-0.51$ \\
$0.56-1.70$ \\
$2.6-24.9$ \\
$3.89-6.1$ \\
\hline
\end{tabular}

\begin{tabular}{c}
\hline PCOS group $(n=4)$ \\
\hline $27.5 \pm 1.29$ \\
$21 \pm 0.81$ \\
$7.54 \pm 0.77$ \\
$16.09 \pm 5.28$ \\
$12.46 \pm 2.52$ \\
$12.32 \pm 5.61$ \\
$59.25 \pm 18.12$ \\
$0.59 \pm 0.17$ \\
$0.845 \pm 0.11$ \\
$2.32 \pm 0.73$ \\
$20.89 \pm 7.79$ \\
$5.51 \pm 0.54$
\end{tabular}

\begin{tabular}{c}
\hline Control group $(n=4)$ \\
\hline $28.25 \pm 1.27$ \\
$21.5 \pm 1.29$ \\
$5.24 \pm 041$ \\
$5.51 \pm 1.11$ \\
$5.61 \pm 0.65$ \\
$13.85 \pm 4.59$ \\
$47.75 \pm 13.94$ \\
$0.46 \pm 0.13$ \\
$0.41 \pm 0.08$ \\
$0.89 \pm 0.25$ \\
$14.84 \pm 2.31$ \\
$4.98 \pm 0.22$ \\
\hline
\end{tabular}

\begin{tabular}{c}
\hline $\boldsymbol{P}$ value \\
\hline 0.660 \\
0.541 \\
0.002 \\
0.025 \\
0.010 \\
0.688 \\
0.356 \\
0.270 \\
0.001 \\
0.024 \\
0.220 \\
0.144 \\
\hline
\end{tabular}

10 ng of tRNA was reverse transcribed with a miRCURY LNA RT Kit (\#339340, Qiagen). The housekeeping gene GAPDH was used for mRNA normalization, and U6 was used for miRNA normalization. The primer sequences used were listed in Table 1. Three independent qPCR runs were analyzed and target gene expression was normalized to the expression of the appropriate housekeeping gene. Furthermore, the obtained Ct values were analyzed using the 2- $\Delta \Delta \mathrm{Ct}$ method (Livak \& Schmittgen 2001).

\section{Exosomal miRNA sequencing and sequence analysis}

The miRNA expression profile was determined in an Ion Torrent PGMTM sequencer (Life Technologies). A TruSeq Small RNA Sample Preparation Kit was used to construct small RNA libraries that preserved strand information. MiRWalk (http://mirwalk.uni-hd.de) and miRBase (http:// www.mirbase.org) were used to analyze the miRNA sequences. In addition, gene ontology (GO) and pathway
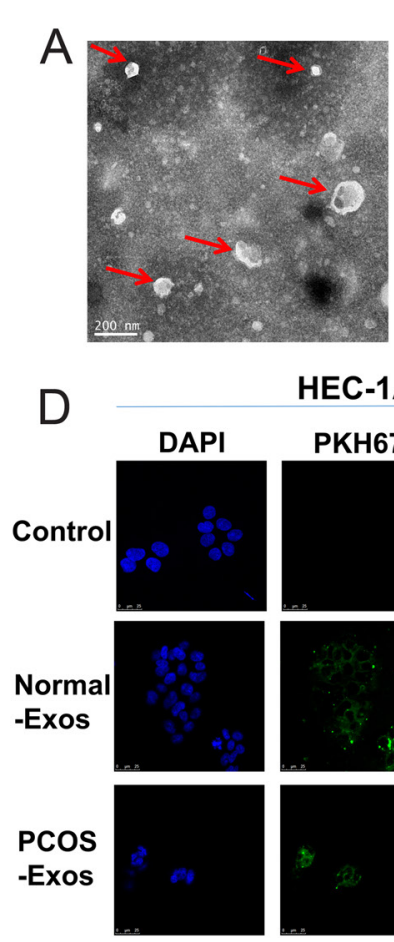

HEC-1A
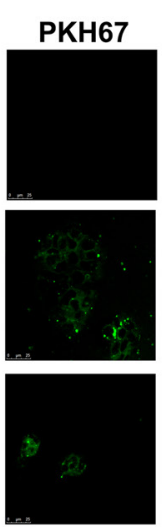

B

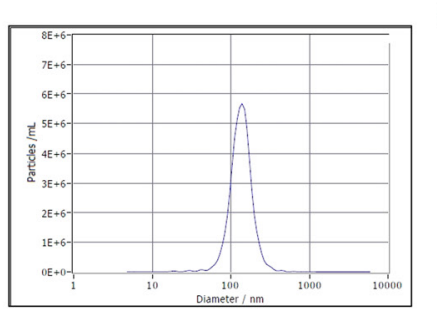

Ishikawa
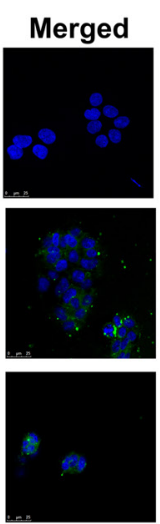

C

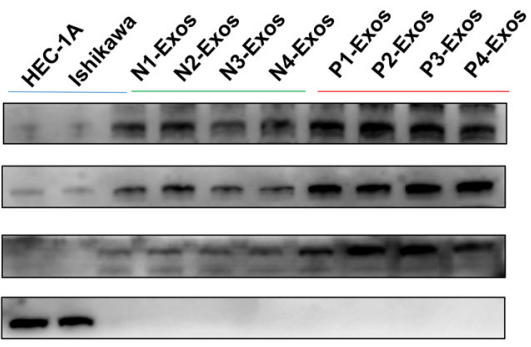

Alix

CD63

Tsg101

Calnexin

Figure 2

(A) PCOS serum-derived exosomes visualized by electron microscopy (scale bar, $200 \mathrm{~nm}$ ). (B) NanoSight analysis of exosomes isolated from the serum of patients with PCOS. (C) Surface markers of exosomes (CD63, HSG101, and Alix) and a negative marker of exosomes (Calnexin), as assessed by Western blotting. (D) PKH67-labeled exosomes from patients with PCOS and normal controls were incubated with the indicated EC cells (scale bar, $25 \mu \mathrm{m}$ ). (E) EC cells treated with PCOS-exosomes revealed prolonged cell migration in an RTCA assay (Mean \pm S.D., $* P<0.05$, ANOVA).

E
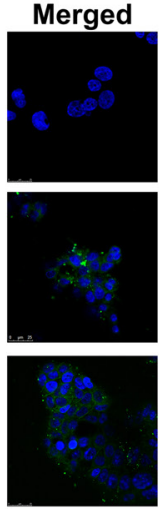

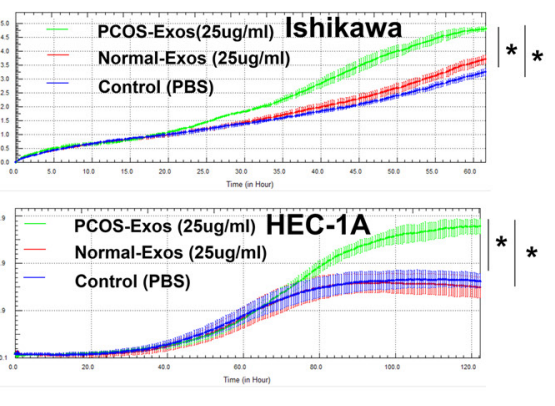


analyses were performed to identify miRNA-related genes, pathways and GO terms based on sequencing data sets.

\section{miR-27a-5p mimics and inhibitor transfection}

HEC-1A and Ishikawa cells $\left(2 \times 10^{5}\right)$ were transiently transfected with $50 \mathrm{nM}$ miR-27a-5p mimics, on negative control (On-NC), miR-27a-5p inhibitor, and Off-NC respectively (RiboBio, Guangzhou, China) for $72 \mathrm{~h}$. Cells were transfected using Lipofectamine 3000 reagent (Life Technologies Corporation) following the manufacturer's instructions. The primers used for the miR-27a-5p On-NC, mimics, Off-NC, and miR-27a-5p inhibitor are shown in Table 1.

\section{Luciferase reporter assay}

We used the TargetScan (http://www.targetscan.org) and miRanda (http://miranda.org) algorithms to screen the potential targets of miR-27a-5p. Fragments of the SMAD4 3'UTR containing the wild-type (WT) or mutant (Mut) predicted binding site for miR-27a-5p were subcloned into the pmirGLO (RiboBio). 293T, Ishikawa, and HEC-1A cells were seeded in 24-well plates. MiR-27a-5p mimics or NC sequences (RiboBio) were cotransfected with pmirGLOSMAD4 WT or pmirGLO-SMAD4 Mut. After $48 \mathrm{~h}$ of transfection, cells were harvested and lysed. Next, firefly luciferase and Renilla luciferase substrates were added to measure the luciferase activity using the Dual-Glo Luciferase Reporter Assay System (Promega). The results of the luciferase assays were analyzed according to the manufacture's instructions.

\section{Western blot analysis}

Ishikawa and HEC-1A cells were trypsinized on ice for $30 \mathrm{~min}$ in RIPA buffer (50 mM Tris-HCl, $150 \mathrm{mM} \mathrm{NaCl}$, $1 \%$ NP-40, $0.5 \%$ sodium deoxycholate and $0.1 \%$ SDS) supplemented protease and phosphatase inhibitors and were then centrifuged at $14,000 \boldsymbol{g}$ for $20 \mathrm{~min}$. Protein lysate was gently sucked up into clean Eppendorf (EP) tubes and its concentration was quantified using the BCA method with a standard protein curve. Gel electrophoresis was used for protein separation. Membranes were incubated overnight at $4^{\circ} \mathrm{C}$ with primary antibodies. Primary antibodies against the following proteins were used: Alix,
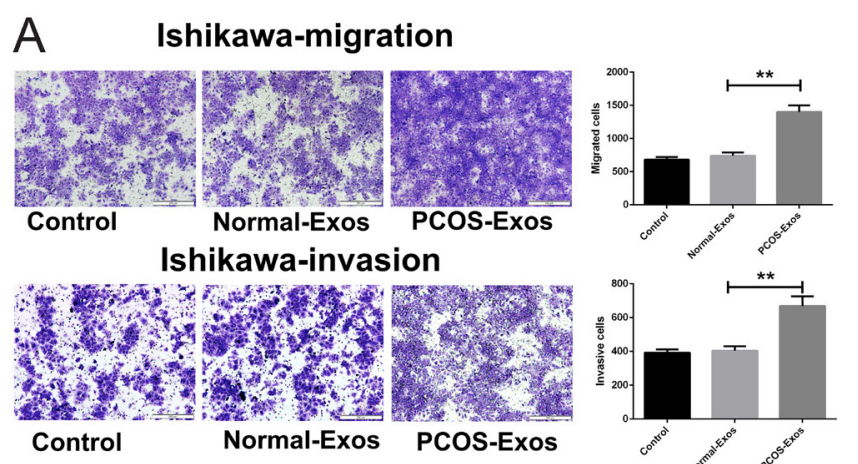

\section{B HEC-1A-migration}
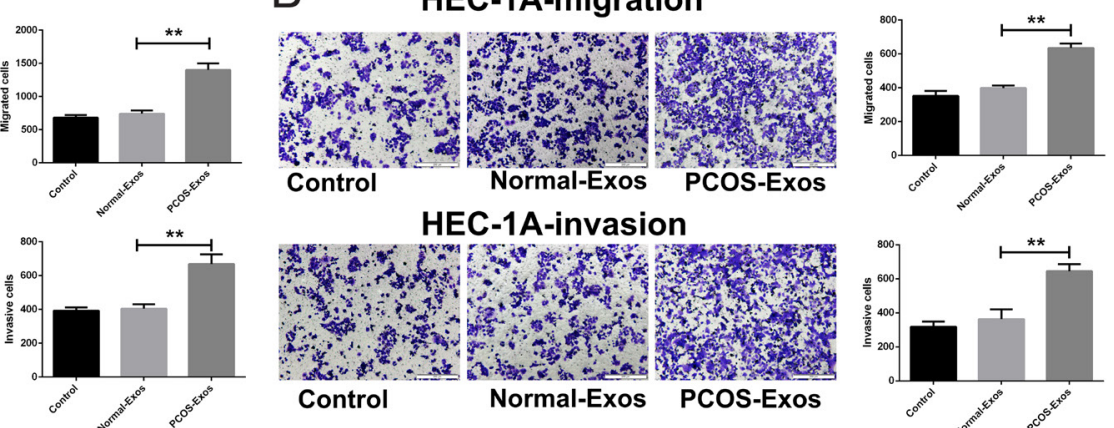

C Ishikawa-scratch wound assay

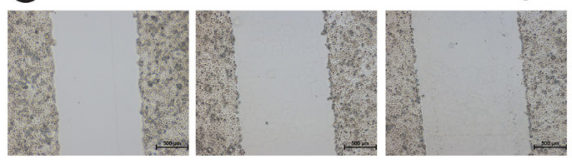

Control-Oh Normal-exos-0h Pcos-exos-0h
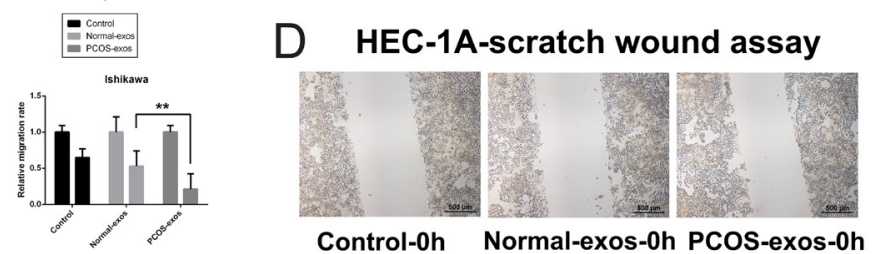

Control-0h Normal-exos-0h PCOS-exos-0h
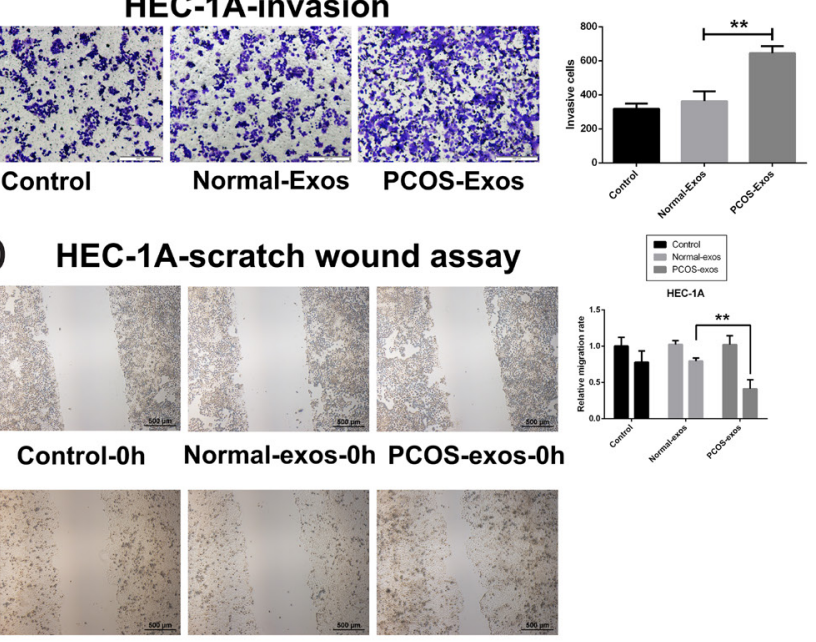

Control-24h Normal-exos-24h PCOS-exos-24h

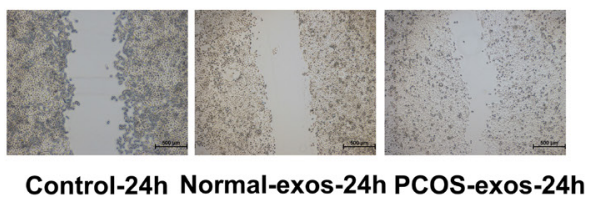

Figure 3

Serum exosomes from PCOS patients promoted EC cell lines migration and invasion. (A and B) Migration and invasion assays of EC cell lines incubated with exosomes from patients with PCOS or normal controls for 48 h (PCOS group: $n=4$; control group: $n=4)$, Mean \pm S.D. **P<0.01, Student's $t$-test. (C and D) Wound-healing assay of EC cell lines incubated with exosomes from PCOS patients or normal controls for $24 \mathrm{~h}$ (PCOS group: $n=4$; control group: $n=4)$, mean \pm S.D., $* * P<0.01$, Student's $t$-test. 


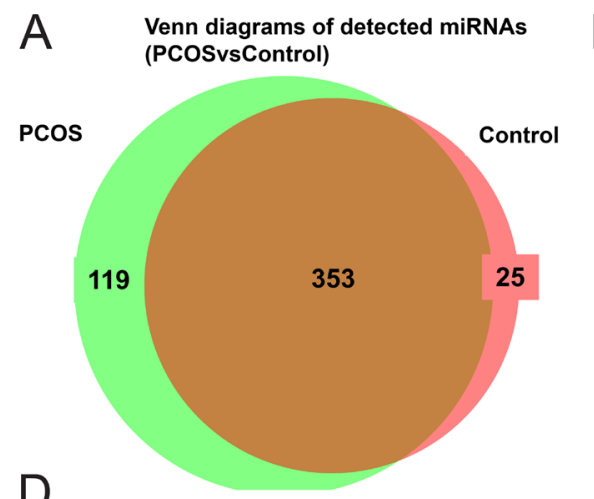

D

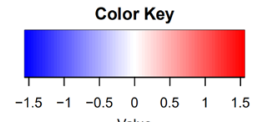

Value
B

Differentially expressed mirnas in different groups

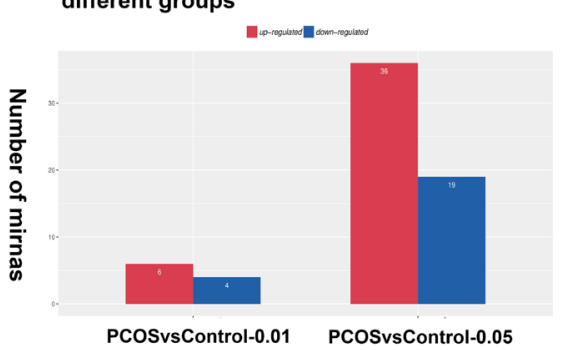

PCOSvsControl $\mathrm{p}<0.01$

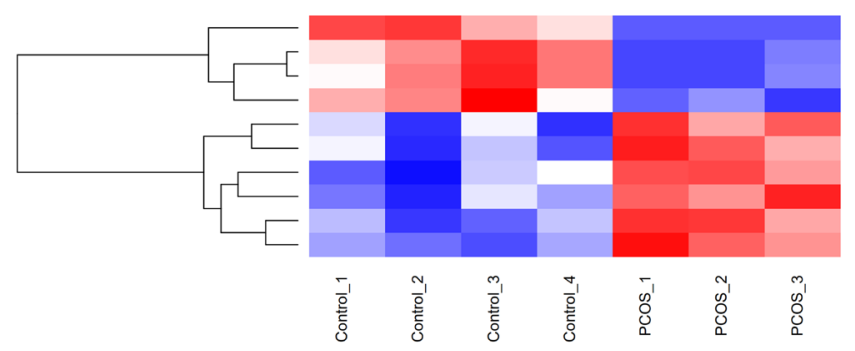

hsa-miR-5189-5p_L-2 hsa-miR-19a-3p_R-2 hsa-miR-19b-3p_R-1 hsa-miR-375-3p hsa-miR-450a-1-3p hsa-miR-2355-3p_L-2R+2 hsa-miR-542-3p hsa-miR-590-3p hsa-mir-590-3p hsa-miR-941
C PCOSvsControl Fold change $>|1.2|, P<0.05$

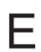

Fold change $>|1.2|, P<0.05$
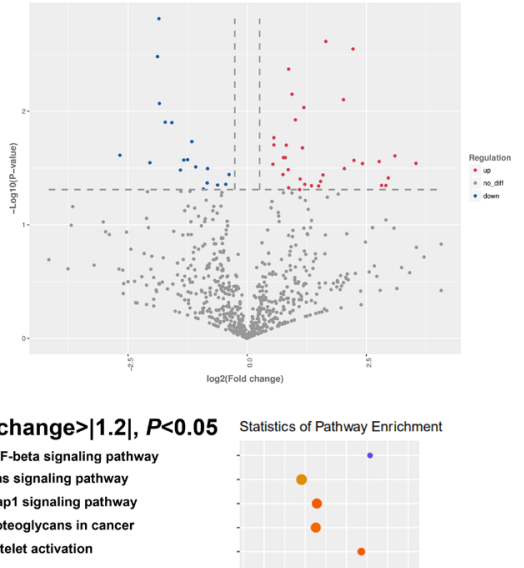
Ras signaling pathway
Raphatheta signaling pathw Rap1 signaling pathway Proteoglycans in cancer Platelet activation PI3K-Akt signaling pathway Phospholipase D signaling pathway Phosphatayinositols

E Phosplatdylnowiong system Pathways in cancer

ते oxyoch oignamg pa Insulin resistance Fc gamma R-mediated phagocytosis Endocytosis Choline metabolism in cancer Cellular senescence Calclum signaling pat AGE-RAGE sinaling pathway in diabetic complicatio Adherens junction

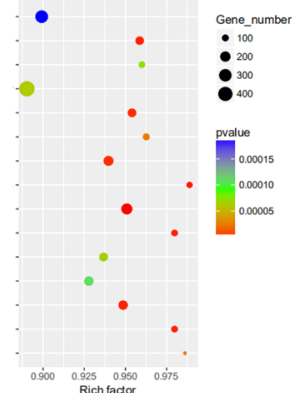

\section{Figure 4}

Cluster analysis of differentially expressed exosomal miRNAs isolated from serum from PCOS patients and normal controls. (A) Venn diagrams of miRNAs detected in the PCOS and normal groups. (B) Bar plot of differentially expressed miRNAs in the two groups $(P<0.05)$. (C) Volcano plot of differentially expressed miRNAs in the two groups ( $|F C|>1.2, P<0.05)$. (D) Heat map of differentially expressed miRNAs in the two groups $(|F C|>1.2, P<0.01)$. (E) KEGG enrichment scatter plots of mRNAs targeted by the differentially expressed miRNAs.

1:1000 (ab117600); CD63, 1:1000 (ab59479); TSG101, 1:1000 (ab83); Calnexin, 1:1000 (CST\#2679); GAPDH, 1:1000 (ab8245); and SMAD4, 1:1000 (CST\#46535).

\section{Statistical analysis}

Values were presented as the means \pm s.D. The statistical significance of the results from three independent assays was evaluated by Student's t-test and one-way analysis of variance. ${ }^{*} P<0.05,{ }^{* *} P<0.01$, and ${ }^{* * *} P<0.001$ were considered as significant.

\section{Results}

\section{PCOS serum exosomes were taken up by EC cells and promoted migration and invasion}

Exosomes were purified from the normal controls $(n=4)$ and PCOS participants' $(n=4)$ serum. The mean age was $27.5 \pm 1.29$ years for the normal controls and $28.25 \pm 1.27$ years for the PCOS participants $(P=0.66)$. The normal controls had a mean body mass index (BMI) of $21 \pm 0.81$, while PCOS patients had a mean BMI of $21.5 \pm 1.29 \mathrm{~kg} / \mathrm{m}^{2}$ $(P=0.541)$. Selected clinical characteristics are shown in
Table 2. We verified the size of serum-derived exosomes isolated from normal controls and PCOS participants using nanoparticle tracking analysis. All vesicles were within the $50-150 \mathrm{~nm}$ size range (Fig. 2A). The typical morphology of the isolated exosomes was also evaluated by transmission electron microscopy (Fig. 2B). The expression of Alix, TSG101, and CD63 but not Calnexin were assessed in the isolated exosomes (Fig. 2C). Exposure of the EC cell lines, Ishikawa and HEC-1A to serum exosomes $(25 \mu \mathrm{g} / \mathrm{mL})$ from normal controls and PCOS patients resulted in positive punctate fluorescent puncta in the cytoplasm (Fig. 2D). EC cells treated with PCOS patients-derived exosomes also revealed an increased cell migration and invasion (Figs $2 \mathrm{E}$ and $3 \mathrm{~A}, \mathrm{~B}, \mathrm{C}, \mathrm{D}$ ) although proliferation was not greatly affected (data not shown).

\section{Differential expression of miRNAs in serum exosomes derived from normal controls and PCOS patients}

To decipher the mechanisms responsible for the proaggressive behavior of PCOS patient-derived exosomes, we analyzed their miRNA content by miRNA sequencing. 
We detected 472 mature miRNAs in the PCOS group and 378 mature miRNAs in the normal control group (Fig. 4A). Statistical analysis of the miRNA sequencing data revealed that the levels of 55 mature miRNAs in serum exosomes from the PCOS group differed significantly from those in serum exosomes from the normal control group $(P<0.05$, $|\mathrm{FC}|>1.2$ ) (Fig. $4 \mathrm{~B}$ and $\mathrm{C}$ ). The heat map showed the differentially expressed miRNAs satisfying $P<0.01$ (Fig. 4D). Moreover, we found that these 55 differentially expressed mature miRNAs putatively target 16,702 mRNAs via both the TargetScan and miRanda databases at the same time. Among the top-ranked canonical pathways in which these mRNAs are involved were pathways in cancer $\left(P=6.05 \times 10^{-5}, 528\right.$ of 7263 predicted molecules targeted $)$ and the PI3K-Akt signaling pathway $\left(P=1.85 \times 10^{-4}, 347\right.$ of 7263 predicted molecules targeted) (Fig. 4E).

Next, we attempted to validate the differences in expression between normal control $(n=10)$ and PCOS $(n=30)$ patient-derived serum exosomes for eight miRNAs (i.e. miR-590-3p, miR-27a-5p, miR-941, miR-2355-3p, miR-542-3p, miR-375a-3p, miR-19a-3p, and miR-19b-3p) that obviously differed according to the miRNA sequencing results. We confirmed significant upregulation of miR-590-3p and miR-27a-5p and downregulation of miR-375a-3p and miR-19b-3p (Fig. 5A) in serum exosomes from PCOS patients relative to their expression in serum exosomes from normal controls.

\section{miR-27a-5p promotes EC cells migration and invasion}

We further explored the biological effects of miR-27a-5p on EC cells phenotypes including cell migration and invasion. Transfection of Ishikawa and HEC-1A cells with the miR-27a-5p mimics resulted in increased of $\sim 60$ and $\sim 40 \%$, respectively, in wound-healing ability compared with transfection with the NC (Fig. 5C). Consistent with the results shown in Fig. 5C, the Matrigel invasion assay and migration assay showed that the numbers of invaded cells and migrated cells were significantly increased in Ishikawa and HEC-1A cells transfected with miR-27a-5p mimics compared with EC cells transfected with On-NC
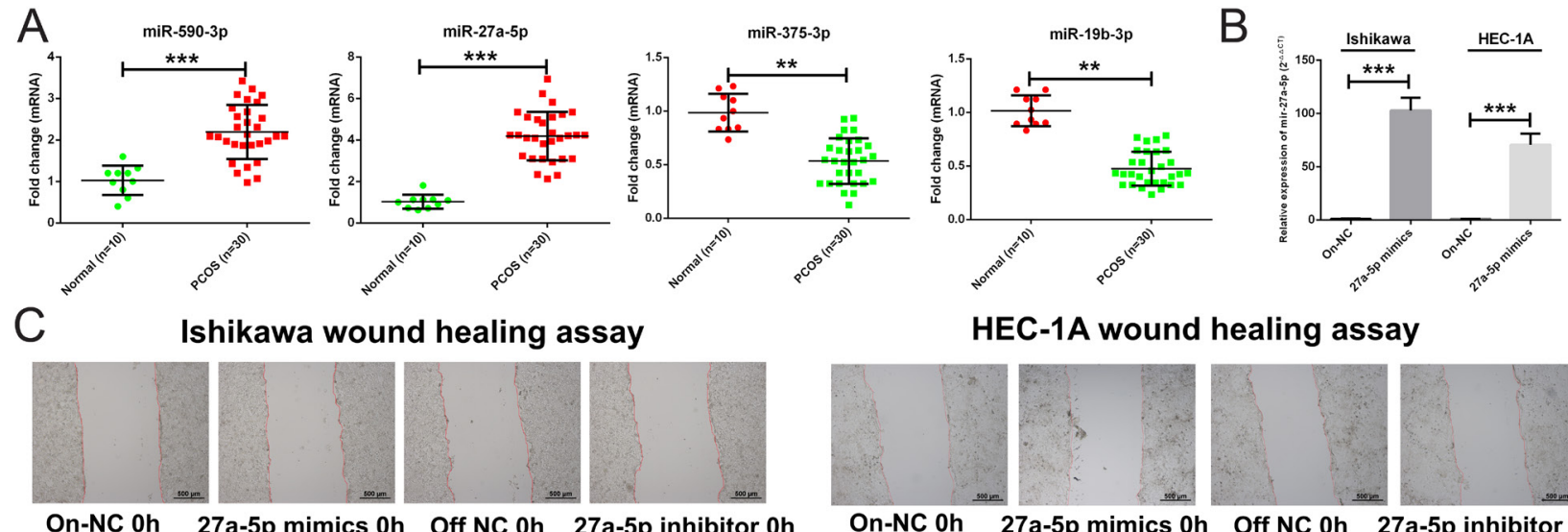

Ishikawa wound healing assay

HEC-1A wound healing assay
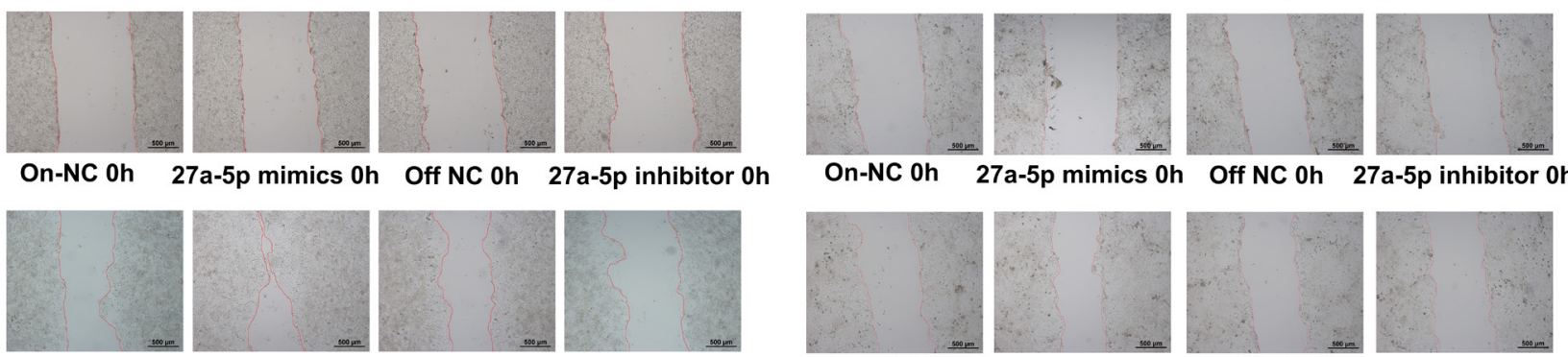

On-NC 24h 27a-5p mimics 24 h Off NC $24 \mathrm{~h} 27 \mathrm{a}-5 \mathrm{p}$ inhibitor $24 \mathrm{~h}$
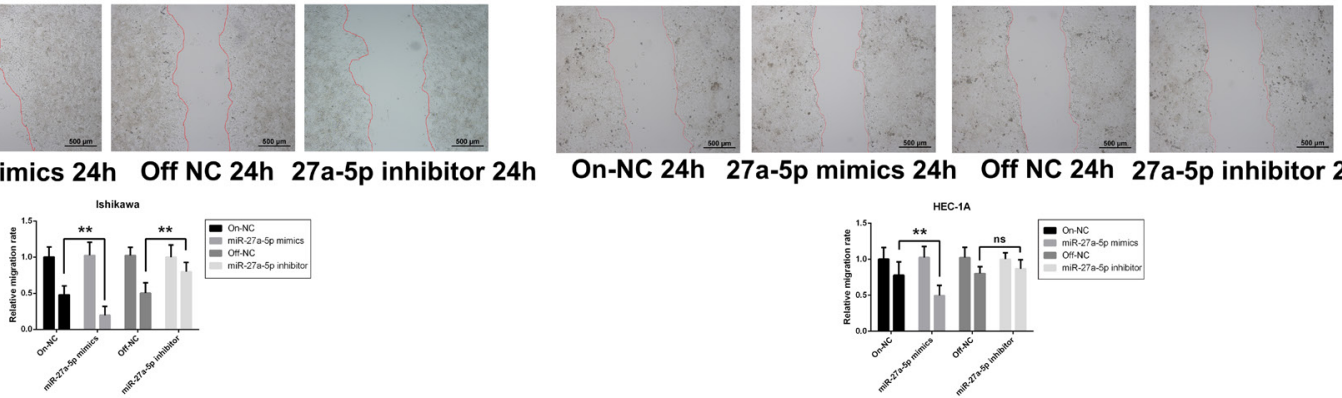

On-NC 24h 27a-5p mimics 24h Off NC 24h 27a-5p inhibitor 24h

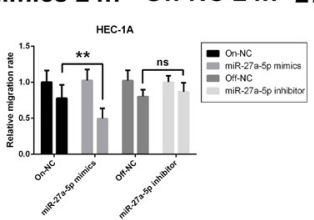

\section{Figure 5}

miR-27a-5p was carried by PCOS serum-derived exosomes. The migration and invasion of Ishikawa and HEC-1A cells was affected by miR-27a-5p mimics or inhibitor transfected. (A) Verification of miR-590-3p, miR-375-3p, miR-27a-5p, and miR-19b-3p in the serum exosomes from PCOS patients and normal controls, mean \pm s.D., $* * P<0.01, * \star * P<0.001$, Student's $t$-test. (B) Expression of miR-27a-5p in Ishikawa and HEC-1A cells transfected with the miR-27a-5p mimics or NC, Mean \pm S.D., $* \star \star P<0.0001$, Student's $t$-test. (C) A wound-healing assay was conducted in miR-27a-5p mimics- or inhibitor-transfected Ishikawa and HEC-1A cells. The migration distance was measured at 0 and $24 \mathrm{~h}$ after the cells were scratched. The results were shown as mean \pm S.D. of three independent experiments. ${ }^{*} P<<0.01$, Student's $t$-test; $n s$, nonsignificant. 

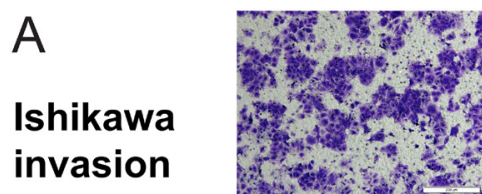

On-NC

\section{Ishikawa migration}

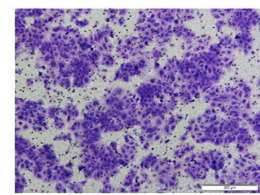

On-NC

B

\section{HEC-1A} invasion

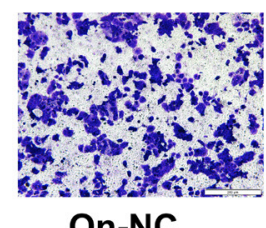

On-NC

HEC-1A
migration

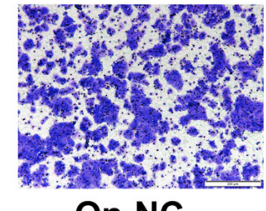

On-NC

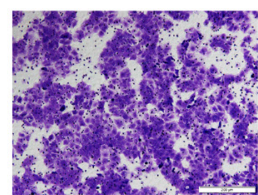

27a-5p mimics

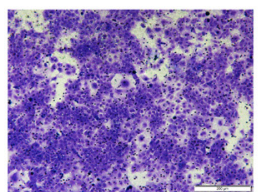

27a-5p mimics

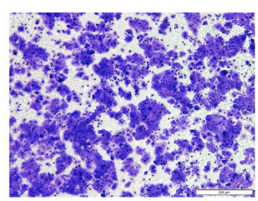

27a-5p mimics

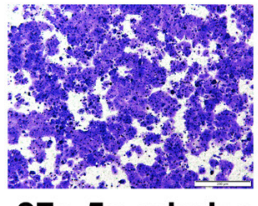

27a-5p mimics

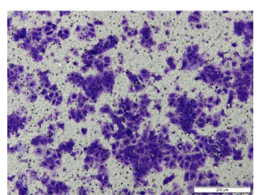

Off-NC

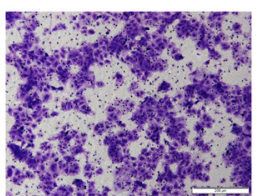

Off-NC

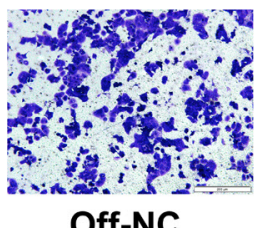

Off-NC

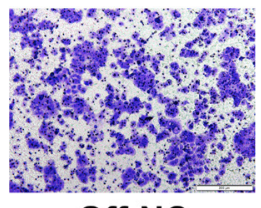

Off-NC

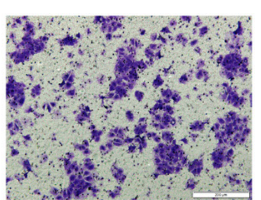

27a-5p inhibitor
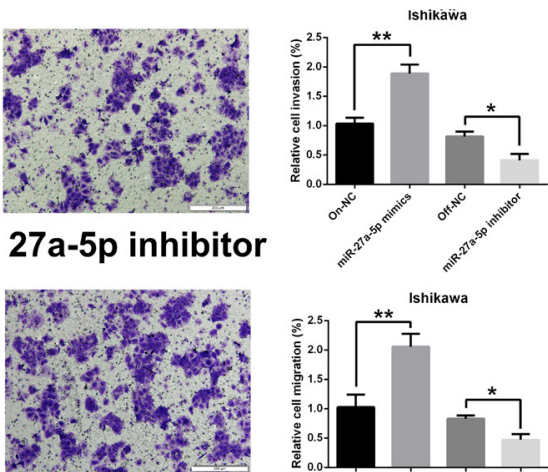

27a-5p inhibitor
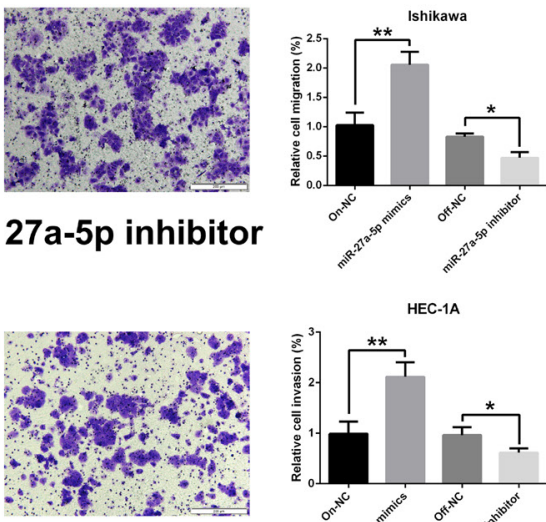

27a-5p inhibitor
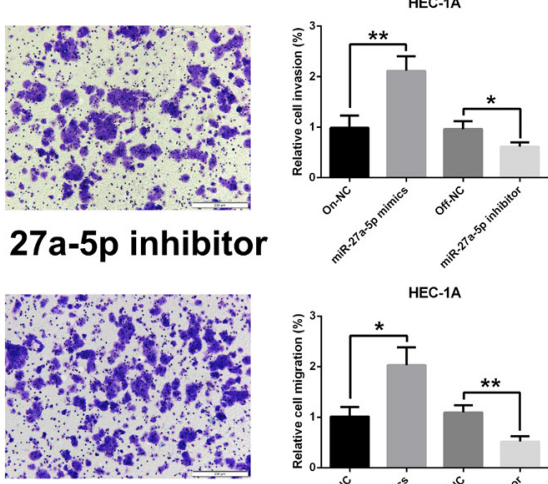

27a-5p inhibitor

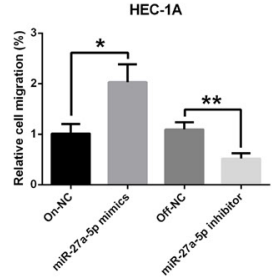

\section{Figure 6}

The migration and invasion of Ishikawa and HEC-1A cells was affected after miR-27a-5p mimics or inhibitor transfection. (A) Migration and invasion assays were conducted in miR-27a-5p mimics- or inhibitor-transfected Ishikawa cells. The migration and invasion rates were shown as the mean \pm S.D. of three independent experiments. ${ }^{*} P<0.05,{ }^{*} * P<0.01$, Student's $t$-test. (B) Migration and invasion assays were conducted in miR-27a-5p mimics- or inhibitor-transfected Ishikawa cells. The migration and invasion rates were shown as the mean \pm S.D. of three independent experiments. ${ }^{\star} P<0.05$, $\star * P<0.01$, Student's $t$-test.

(Fig. 6A and B). In addition, compared with Off-NC, the miR-27a-5p inhibitor significantly suppressed wound healing (Fig. 5C) and decreased migration and invasion in the EC cell lines (Fig. 6A and B). These results indicate that miR-27a-5p has an essential role as a potential oncogene in EC by promoting cell migration and invasion.

\section{PCOS serum-derived exosomal miR-27a-5p} promotes EC cells migration and invasion

We have already shown that PCOS serum-derived exosomes and miR-27a-5p mimics promote EC cells migration and invasion. Whether PCOS serum-derived exosomal miR-27a-5p promotes EC cells migration and invasion was further validated. mir-27a-5p expression was increased by almost four-fold in EC cells treated with PCOS exosomes (Fig. 7A). We further explored the biological effects of PCOS exosomes combined with miR-27a-5p inhibitor. EC cells treated with PCOS exosomes and simultaneously transfected with miR-27a-5p inhibitor exhibited decreased migration $(\sim 50 \%)$ and invasion $(\sim 45 \%)$ rates compared with cells treated with PCOS exosomes alone (Fig. 7B). These results indicate that PCOS serum-derived exosomal miR-27a-5p may promote EC cells migration and invasion.

\section{SMAD4 is a potential direct target of miR-27a-5p}

According to online biological information analysis software (TargetScan and miRanda), SMAD4 is speculated to be a target gene of miR-27a-5p. To support the hypothesis that miR-27a-5p regulates SMAD4 gene expression in EC cells, we first examined SMAD4 mRNA and protein expressions in Ishikawa and HEC-1A cell lines transfected with the miR-27a-5p mimics or inhibitor. The results revealed that overexpression of miR-27a-5p significantly 
A

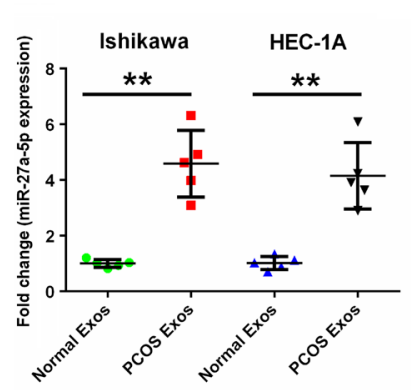

C

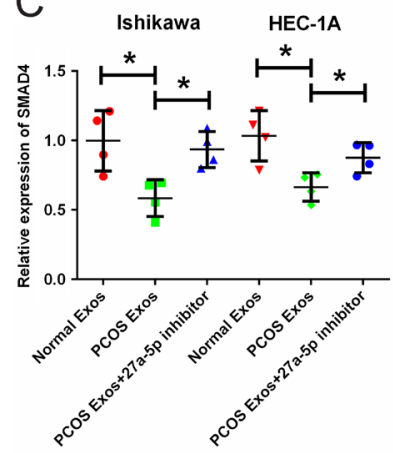

B
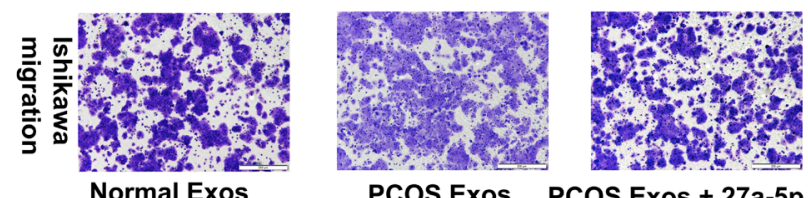

PCOS Exos

PCOS Exos + 27a-5p inhibitor

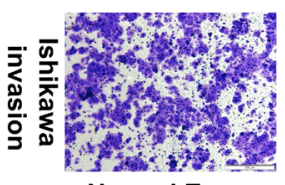

Normal Exos

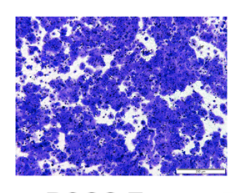

PCos Exos

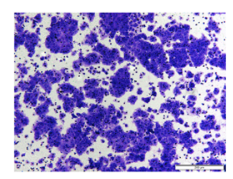

PCOS Exos + 27a-5p inhibitor
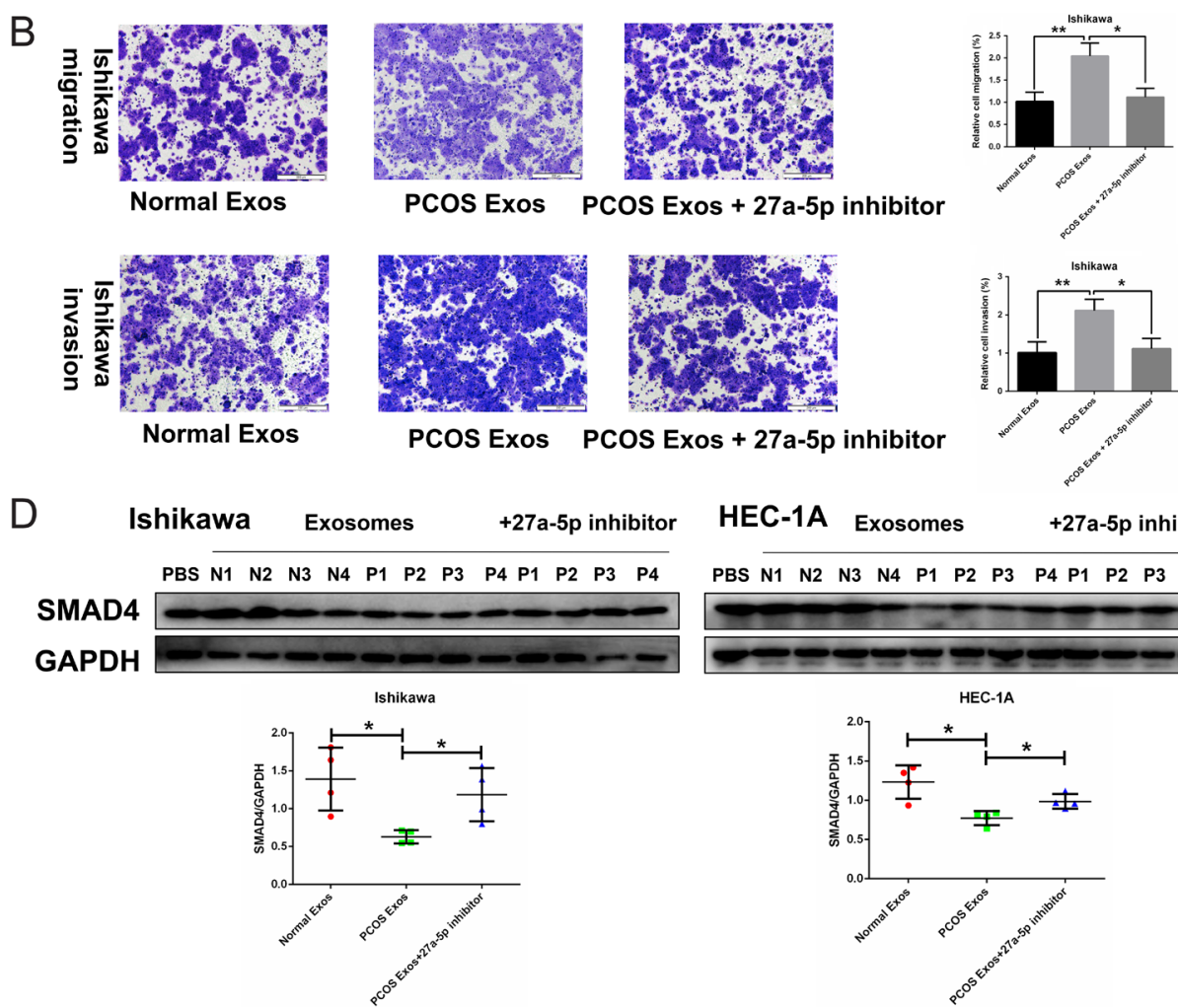

HEC-1A Exosomes +27a-5p inhibitor
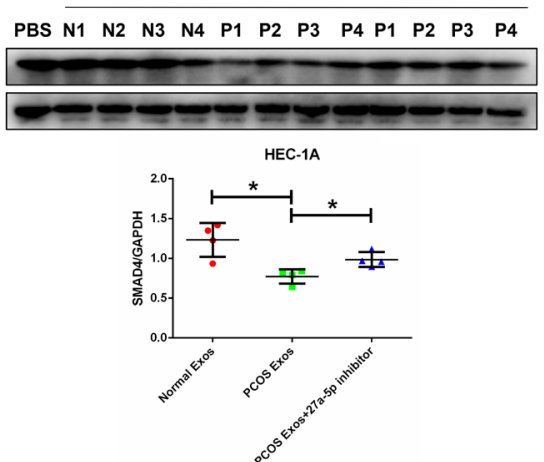

Figure 7

PCOS serum-derived exosome-induced migration and invasion activity was partially rescued by the miR-27a-5p inhibitor. (A) miR-27a-5p expression in Ishikawa and HEC-1A cell lines was detected while adding to serum exosomes derived from normal controls or PCOS patients. EC cells treated with PCOS-exosomes revealed increased miR-27a-5p expression (mean \pm s.D., $* \star P<0.01, * \star * P<0.001$, Student's $t$-test). (B) Migration and invasion assays were conducted in normal controls exosomes, PCOS exosomes or inhibitor-transfected Ishikawa cells. The migration and invasion rate were shown as mean \pm S.D. of three independent experiments. ${ }^{*} P<0.05, * \star P<0.01$, Student's $t$-test. (C) EC cells treated with PCOS-exosomes decreased the SMAD4 mRNA expression, EC cells treated with PCOS exosomes and miR-27a-5p inhibitor rescued the SMAD4 mRNA expression. Mean \pm S.D., $* P<0.05$, Student's $t$-test. (D) EC cells treated with PCOS exosomes decreased the SMAD4 protein expression. EC cells treated with PCOS exosomes and miR-27a-5p inhibitor rescued the SMAD4 protein expression. Results were presented as the mean \pm s.D. ${ }^{\star} P<0.05,{ }^{*} P<<0.01$, Student's $t$-test. GAPDH was used as the loading control.

decreased the mRNA and protein expression levels of SMAD4, whereas inhibition of miR-27a-5p significantly increased these expression levels of SMAD4 (Fig. 8A and $\mathrm{B})$. In addition, we further tested the effect of PCOS serum-derived exosomes on SMAD4 mRNA and protein expression. The results showed that SMAD4 mRNA and protein expressions were decreased upon treatment with PCOS exosomes compared with normal control exosomes. Transfected with miR-27a-5p inhibitor in EC cells combined with PCOS serum-derived exosomes treatment showed that rescued the decreased SMAD4 mRNA and protein expression (Fig. 7C and D). Furthermore, to demonstrate a direct interaction between the SMAD4 3'UTR and miR-27a-5p, we cloned the WT SMAD4 3'UTR region (predicted to interact with miR-27a-5p) into a luciferase reporter vector. We also cloned a SMAD4 3'UTR fragment with mutation of the complementary bases (Fig. 9A). Luciferase activity was reduced by $\sim 45 \%$ in cells were co-transfected with miR-27a-5p ( $P<0.05$, Fig. 9B).
As expected, luciferase activity suppression was significantly abrogated when the interaction between miR-27a-5p and its target 3'UTR was disrupted in cells transfected with SMAD4 3'UTR Mut (Fig. 9).

\section{Discussion}

Women with PCOS have a 2.7-fold increased risk for developing EC. The link between PCOS and EC involves obesity, dyslipidemia, and prolonged endometrial exposure to unopposed estrogen due to anovulation (Dumesic \& Lobo 2013). Emerging studies have shown that exosomes or nanovesicles allow the transfer of proteins, lipids, and nucleic acids between cells. Exosomes secreted by most cell types can be taken up by host cells, suggesting that the tumor microenvironment plays an essential role in tumor development (Kahlert \& Kalluri 2013). In this study, we showed that serum exosomes in PCOS can be taken up by EC cells, leading to increased 

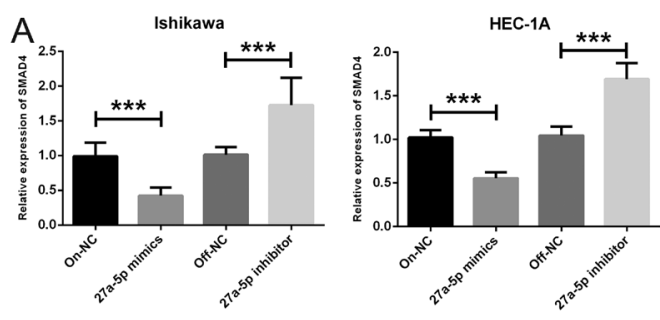

B
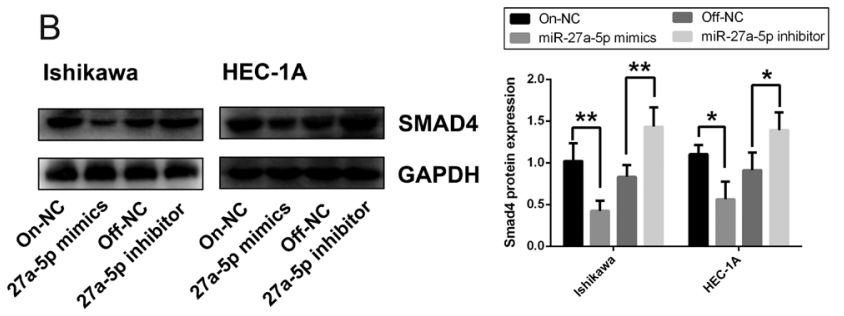

\section{Figure 8}

Expression levels of SMAD4 mRNA and protein in recipient Ishikawa and HEC-1A cells after miR-27a-5p mimics or inhibitor transfection. ( $A$ and $B$ ) miR-27a-5p overexpression in Ishikawa and HEC-1A cells decreased the SMAD4 mRNA and protein levels. Results were presented as mean \pm S.D. $\star P<0.05, * * P<0.01$, and $* * * P<0.0001$, Student's $t$-test. GAPDH was used as the loading control.

migration and invasion, which indicates the importance of serum exosomes in the malignant progression of EC in the context of PCOS. The transfer of exosome-derived unique miRNA to recipient cells has been proposed as an alternative mechanism that involves cell-to-cell communication (Kosaka et al. 2013). Li et al. (2019a) found that CAF-mediated EC progression was related to the loss of miR-148b in the CAF-derived exosomes. In addition, bone marrow mesenchymal stem cell (BMSC)derived exosomal miR-126-3p was proven to inhibit pancreatic cancer development by targeting ADAM9
(Wu et al. 2019). Lazar et al. (2016) also proposed a novel mechanism linking obesity and cancer. These researchers proved that adipose tissue-derived exosomes containing fatty acid oxidation products promoted melanoma aggressiveness. Collectively, these findings indicated that miRNAs may be secreted and then delivered into recipient cells via the exosome pathway. The cellular functions of the recipient cells may be altered by the exosomal miRNAs via modulation of the expression of these miRNAs' target genes. In our study, the specific mechanism by which miR-27a-5p transferred via serum exosomes from patients with PCOS influenced the biological function of EC cell lines was identified. Mir-27a-5p shuttled by serum exosomes from the patients with PCOS increased the migration and invasion of Ishikawa and HEC-1A by directly targeting SMAD4. Thus, miR-27a-5p may be a biomarker for the clinical prevention of EC development in the context of PCOS.

miRNAs can be transferred between cancer cells and stromal cells, resulting in abundant downregulation of mRNAs to reprogram the target cell transcriptome (Melo et al. 2014). Initially, the EC cell lines Ishikawa and HEC-1A were treated with exosomes derived from the serum of PCOS patients. Our findings revealed that exosomes promoted progressive behaviors, including the migration and invasion of EC cell lines (Figs 2 and 3). Next, exosomal miRNA sequencing was used to identify differentially expressed miRNAs between serum exosomes from PCOS patients and normal controls. miR-27a-5p expression was increased in the PCOS group compared with the normal control group (Fig. 4). Ishikawa and HEC-1A cell lines transfected with the miR-27a-5p

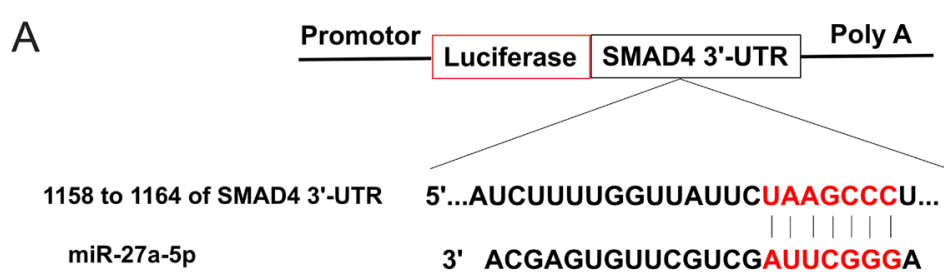

1158 to 1164 of SMAD4 3'-UTR mut $\quad 5 '$ '...AUCUUUUGGUUAUUCAUUCGGGT... B
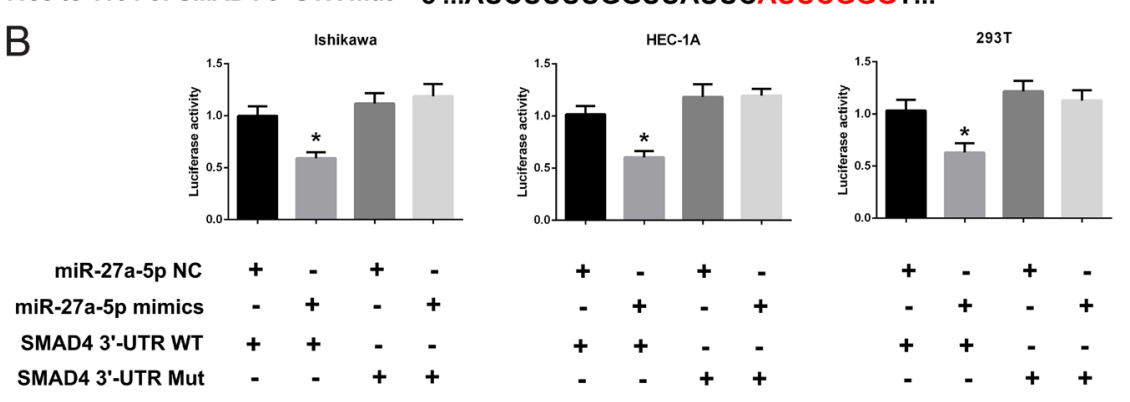

$$
\begin{array}{rrrr}
+ & - & + & - \\
- & + & - & + \\
+ & + & - & - \\
- & - & + & +
\end{array}
$$

\section{Figure 9}

Targeting of SMAD4 by miR-27a-5p was confirmed by a dual luciferase reporter assay. (A) Construction of the SMAD4-3'UTR WTand SMAD4-3'UTR Mut vectors and the active sequence of miR-27a-5p. (B) Luciferase activity in each group of Ishikawa, HEC-1A, and 293T cells, * indicated $P<0.05$ compared with the miR-27a5p-NC + SMAD4-3'UTR, miR-27a-5p-NC + SMAD43'UTR Mut and miR-27a-5p mimics + SMAD4-3'UTR Mut groups. Mean \pm s.D., Student's $t$-test. A full colour version of this figure is available at https:// doi.org/10.1530/JME-19-0159. 
mimics exhibited enhanced migration and invasion. In contrast, these cells transfected with the miR-27a-5p inhibitor exhibited decreased migration and invasion (Figs 5 and 6).

miR-27a was shown to be located on chromosome 19 , and it plays an essential role in tumor development (Li et al. 2019b). MiR-27a could be either a tumor suppressor or an oncomiRNA in several types of cancers, including breast cancer, pancreatic cancer and colon cancer. Moreover, miR-27a is involved in many processes of tumor growth, including cell proliferation, invasion, apoptosis and angiogenesis. Ba et al. (2017) showed that miR-27a promoted the proliferation and invasion of colon cancer cells by targeting SFRP1 through the Wnt/ $\beta$-catenin signaling pathway. Similarly, a study by Sun et al. (2015) showed that miR-27a, which was upregulated in obese patients, promoted proliferation ability in liver cancer by targeting FOXO1. Chae et al. (2017) showed that SMAD2 and SMAD4, the preferred target genes, were inhibited by miR-27a to regulate the TGF- $\beta$ signaling pathway in lung cancer. In contrast, Li et al. (2018) also reported that miR-27a could act as a tumor suppressor in breast cancer. miR-27a was proven to suppress breast cancer cells proliferation and migration by targeting and downregulating TMEM170B. Consistent with this discovery, miR-27a was downregulated and inhibited cell invasion via targeting and negatively regulating KRAS in esophageal squamous cell carcinoma cells (Jiang et al. 2015). Our study also found decreased mRNA and protein expression levels of SMAD4 in Ishikawa and HEC-1A cells transfected with the miR27a-5p mimics, and vice versa (Fig. 6B and C). Moreover, miR-27a-5p suppressed luciferase reporter activity in 293T, Ishikawa, and HEC-1A cells cotransfected with the WT SMAD4-3'UTR but not that with the SMAD4-3'UTR Mut (Fig. 8). Therefore, we showed that SMAD4 was a direct target gene of miR-27a-5p.

Recently, numerous studies have proven the emerging role of exosomal biomarkers in cancer diagnosis and prognosis assessment. Here, our data showed that the level of exosomal miR-27a-5p derived from PCOS patients was relatively higher than that from normal controls. In addition, we verified that transfection of the EC cell lines Ishikawa and HEC-1A with the miR-27a-5p mimics promoted the migration and invasion of these cells via direct targeting of SMAD4. Collectively, our experimental data suggest that quantitative blood testing of PCOS patients for miR-27a-5p levels in circulating exosomes may be useful as a marker for initiating EC preventive treatment.

\section{Declaration of interest}

The authors declare that there is no conflict of interest that could be perceived as prejudicing the impartiality of the research reported.

\section{Funding}

This work was supported by the Shanghai Municipal Education CommissionGaofeng Clinical Medicine Grant (20172003); the National Natural Science Foundation of China (81572836); the Science and Technology Commission of Shanghai Municipality (grant number 16411953500).

\section{References}

Ba S, Xuan Y, Long ZW, Chen HY \& Zheng SS 2017 MicroRNA-27a promotes the proliferation and invasiveness of colon cancer cells by targeting SFRP1 through the Wnt/beta-catenin signaling pathway. Cellular Physiology and Biochemistry 42 1920-1933. (https://doi. org/10.1159/000479610)

Bartel DP 2004 MicroRNAs: genomics, biogenesis, mechanism, and function. Cell 116 281-297. (https://doi.org/10.1016/s00928674(04)00045-5)

Chae DK, Ban E, Yoo YS, Kim EE, Baik JH \& Song EJ 2017 MIR-27a regulates the TGF-beta signaling pathway by targeting SMAD2 and SMAD4 in lung cancer. Molecular Carcinogenesis 56 1992-1998. (https://doi.org/10.1002/mc.22655)

Dumesic DA \& Lobo RA 2013 Cancer risk and PCOS. Steroids 78 782-785. (https://doi.org/10.1016/j.steroids.2013.04.004)

Ha M \& Kim VN 2014 Regulation of microRNA biogenesis. Nature Reviews: Molecular Cell Biology 15 509-524. (https://doi.org/10.1038/ nrm3838)

Huang X, Yuan T, Liang M, Du M, Xia S, Dittmar R, Wang D, See W, Costello BA, Quevedo F, et al. 2015 Exosomal miR-1290 and miR375 as prognostic markers in castration-resistant prostate cancer. European Urology 67 33-41. (https://doi.org/10.1016/j. eururo.2014.07.035)

Irelan JT, Wu MJ, Morgan J, Ke N, Xi B, Wang X, Xu X \& Abassi YA 2011 Rapid and quantitative assessment of cell quality, identity, and functionality for cell-based assays using real-time cellular analysis. Journal of Biomolecular Screening 16 313-322. (https://doi. org/10.1177/1087057110397359)

Jiang Y, Duan Y \& Zhou H 2015 MicroRNA-27a directly targets KRAS to inhibit cell proliferation in esophageal squamous cell carcinoma. Oncology Letters 9 471-477. (https://doi.org/10.3892/ol.2014.2701)

Kahlert C \& Kalluri R 2013 Exosomes in tumor microenvironment influence cancer progression and metastasis. Journal of Molecular Medicine 91 431-437. (https://doi.org/10.1007/s00109013-1020-6)

Kosaka N, Yoshioka Y, Hagiwara K, Tominaga N, Katsuda T \& Ochiya T 2013 Trash or treasure: extracellular microRNAs and cell-to-cell communication. Frontiers in Genetics 4 173. (https://doi.org/10.3389/ fgene.2013.00173)

Kumar B \& Lupold SE 2016 MicroRNA expression and function in prostate cancer: a review of current knowledge and opportunities for discovery. Asian Journal of Andrology 18 559-567. (https://doi. org/10.4103/1008-682X.177839)

Lazar I, Clement E, Dauvillier S, Milhas D, Ducoux-Petit M, LeGonidec S, Moro C, Soldan V, Dalle S, Balor S, et al. 2016 Adipocyte exosomes promote melanoma aggressiveness through fatty acid oxidation: a novel mechanism linking obesity and cancer. Cancer Research 76 4051-4057. (https://doi.org/10.1158/0008-5472. CAN-16-0651)

Li M, Han Y, Zhou H, Li X, Lin C, Zhang E, Chi X, Hu J \& Xu H 2018 Transmembrane protein $170 \mathrm{~B}$ is a novel breast tumorigenesis 
suppressor gene that inhibits the Wnt/beta-catenin pathway. Cell Death and Disease 9 91. (https://doi.org/10.1038/s41419-017-0128-y)

Li BL, Lu W, Qu JJ, Ye L, Du GQ \& Wan XP 2019a Loss of exosomal miR-148b from cancer-associated fibroblasts promotes endometrial cancer cell invasion and cancer metastasis. Journal of Cellular Physiology 234 2943-2953. (https://doi. org/10.1002/jcp.27111)

Li X, Xu M, Ding L \& Tang J $2019 b$ MiR-27a: a novel biomarker and potential therapeutic target in tumors. Journal of Cancer $\mathbf{1 0}$ 2836-2848. (https://doi.org/10.7150/jca.31361)

Liu Y, Gu Y \& Cao X 2015 The exosomes in tumor immunity. OncoImmunology 4 e1027472. (https://doi.org/10.1080/21624 02X.2015.1027472)

Livak KJ \& Schmittgen TD 2001 Analysis of relative gene expression data using real-time quantitative PCR and the 2(-Delta Delta C(T)) Method. Methods 25 402-408. (https://doi.org/10.1006/ meth.2001.1262)

Melo SA, Sugimoto H, O'Connell JT, Kato N, Villanueva A, Vidal A, Qiu L, Vitkin E, Perelman LT, Melo CA, et al. 2014 Cancer exosomes perform cell-independent microRNA biogenesis and promote tumorigenesis. Cancer Cell 26 707-721. (https://doi.org/10.1016/j. ccell.2014.09.005)

Melo SA, Luecke LB, Kahlert C, Fernandez AF, Gammon ST, Kaye J, LeBleu VS, Mittendorf EA, Weitz J, Rahbari N,, et al. 2015 Glypican-1 identifies cancer exosomes and detects early pancreatic cancer. Nature 523 177-182. (https://doi.org/10.1038/nature14581)

Papaioannou S \& Tzafettas J 2010 Anovulation with or without PCO, hyperandrogenaemia and hyperinsulinaemia as promoters of endometrial and breast cancer. Best Practice and Research: Clinical Obstetrics and Gynaecology 24 19-27. (https://doi.org/10.1016/j. bpobgyn.2008.11.010)

Patras L \& Banciu M 2019 Intercellular crosstalk via extracellular vesicles in tumor milieu as emerging therapies for cancer progression. Current Pharmaceutical Design 25 1980-2006. (https://doi.org/10.2174 /1381612825666190701143845)

Sun B, Li J, Shao D, Pan Y, Chen Y, Li S, Yao X, Li H, Liu W, Zhang M,, et al. 2015 Adipose tissue-secreted miR-27a promotes liver cancer by targeting FOXO1 in obese individuals. OncoTargets and Therapy $\mathbf{8}$ 735-744. (https://doi.org/10.2147/OTT.S80945)

Valadi H, Ekstrom K, Bossios A, Sjostrand M, Lee JJ \& Lotvall JO 2007 Exosome-mediated transfer of mRNAs and microRNAs is a novel mechanism of genetic exchange between cells. Nature Cell Biology 9 654-659. (https://doi.org/10.1038/ncb1596)

Wu QB, Sheng X, Zhang N, Yang MW \& Wang F 2018 Role of microRNAs in the resistance of colorectal cancer to chemoradiotherapy. Molecular and Clinical Oncology 8 528-532. (https://doi.org/10.3892/mco.2018.1578)

Wu DM, Wen X, Han XR, Wang S, Wang YJ, Shen M, Fan SH, Zhang ZF, Shan Q, Li MQ, et al. 2019 Bone marrow mesenchymal stem cellderived exosomal MicroRNA-126-3p inhibits pancreatic cancer development by targeting ADAM9. Molecular Therapy: Nucleic Acids 16 229-245. (https://doi.org/10.1016/j.omtn.2019.02.022)

Yanokura M, Banno K, Iida M, Irie H, Umene K, Masuda K, Kobayashi Y, Tominaga E \& Aoki D 2015 MicroRNAs in endometrial cancer: recent advances and potential clinical applications. Excli Journal 14 190-198. (https://doi.org/10.17179/excli2014-590)

Received in final form 5 November 2019

Accepted 11 November 2019

Accepted Manuscript published online 11 November 2019
(C) 2020 Society for Endocrinology Published by Bioscientifica Ltd. Printed in Great Britain 